\title{
Steric Effects on Water Accessability Control Sequence-Selectivity of Radical Cation Reactions in DNA
}

\author{
Charles L. Cleveland, ${ }^{\dagger}$ Robert N. Barnett,${ }^{\dagger}$ Angelo Bongiorno, ${ }^{\dagger}$ Joshy Joseph,,${ }^{\ddagger}$ Chusheng Liu,, \\ Gary B. Schuster, ${ }^{*, \neq}$ and Uzi Landman ${ }^{*, \dagger}$ \\ Schools of Physics, and Chemistry and Biochemistry, Georgia Institute of Technology, Atlanta, Georgia 30332 \\ Received March 17, 2007; E-mail: uzi.landman@physics.gatech.edu; gary.schuster@cos.gatech.edu
}

The discovery that electron holes (radical cations) migrate long distances in DNA has fostered enormous interest in the mechanism of this processes, due in large part to its apparent similarity with oxidative damage in cells. ${ }^{1,2}$ Several models have been proposed to account for the extensive experimental observations in this area. Currently, it is generally accepted that holes migrate long distances through duplex DNA by a series of short hops pausing briefly at guanine-containing sites of relatively low oxidation potential (often GG steps) where they may react irreversibly with $\mathrm{H}_{2} \mathrm{O}$ or $\mathrm{O}_{2}{ }^{3,4}$ The two guanines of the GG steps are not equally reactive. A hallmark of the one-electron oxidation of duplex DNA is that the $5^{\prime}$-guanine of GG steps is typically more reactive than the $3^{\prime}-\mathrm{G}^{5-7}$ This finding has been analyzed computationally. Both ab initio and NDDO-G gas-phase model calculations have been interpreted to indicate that electronic effects make the $5^{\prime}-\mathrm{G}$ of GG steps more easily oxidizable than the $3^{\prime}$-guanine. ${ }^{8,9}$ It was also suggested that the $5^{\prime}-\mathrm{G}$ radical cation is stabilized by electronic overlap with the $\mathrm{N} 7$ nitrogen atom and $\mathrm{O} 6$ oxygen atom of the $3^{\prime}-\mathrm{G}^{10}$ and that the reactivity pattern seen in GG steps is controlled by the identity of adjacent nucleobases. ${ }^{11}$

To assess the influence of electronic structure on the sequence selectivity of guanine oxidation in DNA, we calculated the ionization potentials (IP, evaluated as the difference between the total energies of the neutral and ionized sequences) and the hole spatial distributions of sequences d $\left(5^{\prime}-\mathrm{XGGX}-3^{\prime}\right) / \mathrm{d}\left(3^{\prime}-\mathrm{YCCY}^{\prime} 5^{\prime}\right)$, where $\mathrm{X}=\mathrm{A}, \mathrm{T}, \mathrm{U}$, and $\mathrm{Y}$ is the complementary base. These calculations are quantitatively reliable, ${ }^{12}$ and the calculated and experimentally measured IPs of the individual nucleobases agree. ${ }^{13}$ The calculated IPs for these gas-phase, base-paired quartets ${ }^{14}$ are 4.02, 4.04, and $4.08 \mathrm{eV}$ for $\mathrm{X}=\mathrm{A}, \mathrm{T}$, and $\mathrm{U}$, respectively, which is in the expected order. Most significantly, the hole distribution (Table 1) is remarkably insensitive to X. The hole is similarly delocalized over the XGGX sequences with only a modest preference for the $5^{\prime}-\mathrm{G}$. This finding suggests that electronic factors may not be the primary determinant of the reaction selectivity for GG steps in these cases.

We also carried out detailed classical molecular dynamics (MD)

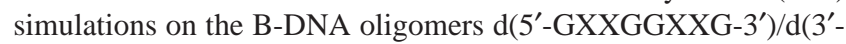
GYYGGYYG-5') that suggest there is an important steric contribution to the preference for reaction at the $5^{\prime}-\mathrm{G}$ in the GG doublets. These simulations ${ }^{15}$ reveal that stacking in these instances is essentially independent of sequence (consistent with X-ray data for DNA and $\mathrm{RNA}^{13}$ ), and yield pair-distribution functions, $g(r ; \mathrm{C} 8)$, between the reactive sites - $\mathrm{C} 8$ of the $5^{\prime}$ - and $3^{\prime}$-guanines and the oxygen atoms of neighboring water molecules. These water molecules lie in a cone-shaped region (half opening angle $30^{\circ}$ ) with the apex at the guanine $\mathrm{C} 8$ atom and extending to the $3^{\prime}$-side (reaction at the $5^{\prime}$-side is blocked sterically by the adjacent base ${ }^{13}$ ).

$\dagger$ School of Physics.

\$ School of Chemistry and Biochemistry.
Table 1. Hole Occupation Fractions and the Ratio of the Hole Density on $5^{\prime}$ and 3' Guanines in GG Steps from Gas-Phase Calculations on Duplex B-DNA Quartets: $5^{\prime}-X G G X-3^{\prime} / 3^{\prime}-Y C C Y-5^{\prime a}$

\begin{tabular}{lccc}
\hline & $X=A$ & $X=T$ & $X=U$ \\
\hline $5^{\prime}-X$ & 0.02 & 0.01 & 0.01 \\
$G$ & 0.50 & 0.53 & 0.51 \\
$G$ & 0.43 & 0.45 & 0.44 \\
$3^{\prime}-X$ & 0.04 & 0.01 & 0.01 \\
$5^{\prime}-G / 3^{\prime}-G$ & 1.16 & 1.18 & 1.16 \\
\hline
\end{tabular}

${ }^{a}$ The hole occupations on the complementary strand YCCY and on the sugar-phosphate backbone are negligible.

This cone is oriented in the "tetrahedral bond direction", that is, that defined by the developing $\mathrm{sp}^{3}$ carbon-oxygen bonding orbital. ${ }^{12}$ Time-averaged calculated $g(r ; \mathrm{C} 8)$ show (Figure 1) that the probability of finding water molecules in the reactive conical sector of the $5^{\prime}-\mathrm{G}$ is essentially sequence invariant. In contrast, the $g(r$; C8) calculated for the $3^{\prime}-\mathrm{G}$ exhibits a strong sequence dependence with the peak of the distribution highest for the $5^{\prime}$-AGGA-3' sequence and lowest for 5'-TGGT-3', with the 5'-UGGU-3' intermediate between the two. The sequence dependence of the water access probability to the $\mathrm{C} 8$ site is due to steric hindrance by the thymine methyl group, which, of course, is absent in uracil. ${ }^{13}$

To assess the validity of this prediction, we undertook a systematic experimental investigation of the one-electron oxidation of DNA oligomers designed specifically to separate steric from electronic effects in the control of reaction selectivity at GG steps. The series of DNA oligomers (Figure 2) was prepared to resolve the affect of neighboring bases on the relative reactivity of the two guanines in GG steps into electronic and steric components. Each duplex contains a covalently linked anthraquinone photosensitizer (AQ) at a $5^{\prime}$-end and is radiolabeled with ${ }^{32} \mathrm{P}$ for PAGE analysis and quantitative phosphorimagery. Each duplex also contains a region of regularly repeating nucleobases. For example, in DNA(1) this region contains the series $\left(5^{\prime}-\mathrm{AGG}-3^{\prime}\right)_{6}$, which generates a string of six AGGA sequences. Similarly, DNA(2) contains six consecutive TGGT sequences and DNA(3) has successive UGGU sequences. These three DNA duplex oligomers have the expected melting behavior and show circular dichroism spectra characteristic of B-form DNA.

The DNA duplex oligomers were irradiated ${ }^{16}$ and then treated with piperidine, which causes strand cleavage at the site of an oxidized guanine. ${ }^{1}$ The samples were analyzed by gel electrophoresis, and the amount of strand cleavage was quantified by phosphorimagery. A typical gel and the phosphorimagery data are shown in Figure 3. As expected for these three DNA duplexes, the amount of strand cleavage at each of the GG steps is the same within experimental error because the rate of hole hopping $\left(k_{\mathrm{hop}}\right)$ is significantly greater than the rate of the irreversible trapping reaction $\left(k_{\text {trap }}\right)$, and thus the distribution of holes among the reaction sites is controlled thermodynamically. ${ }^{3}$ 


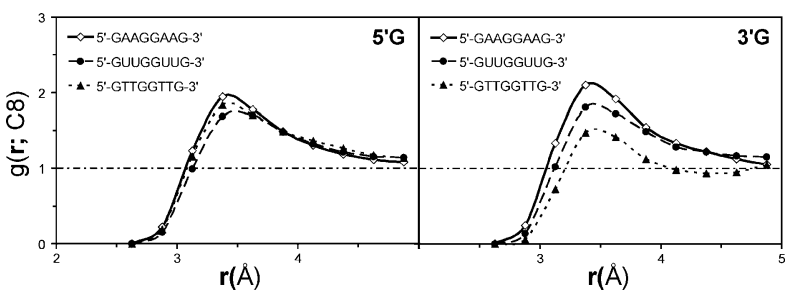

Figure 1. Pair distribution functions, $g(r ; \mathrm{C} 8)$, between the reactive sitesthe $\mathrm{C} 8$ carbon atom of the $5^{\prime}$ - and $3^{\prime}$-guanines - and the oxygen atoms of neighboring water molecules. Results shown for 3 sequences.

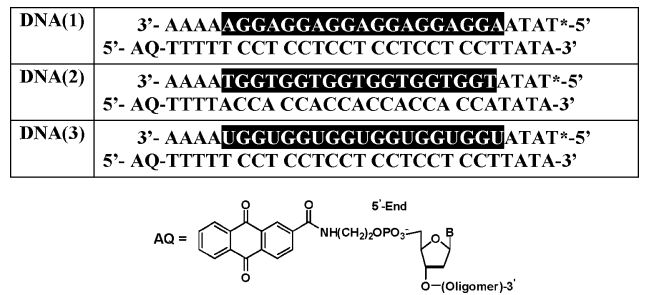

Figure 2. Structures of DNA oligomers used in this work. The $*$ denotes the position of ${ }^{32} \mathrm{P}$ radioactive label.

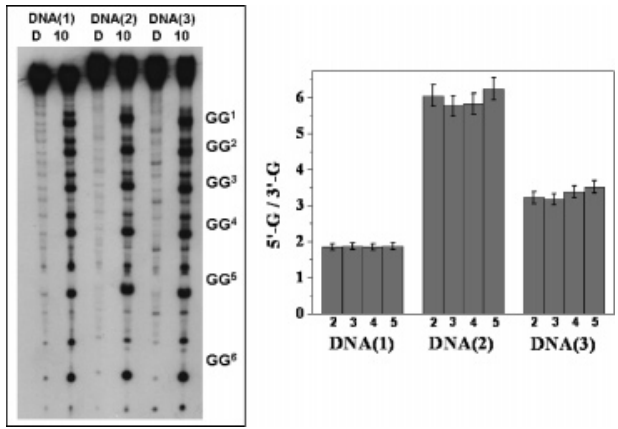

Figure 3. Autoradiogram of PAGE gel following the irradiation of DNA(1) - DNA(3). The graph in the right side shows the ratio of damage for $5^{\prime}-\mathrm{G}$ and $3^{\prime}-\mathrm{G}$ for the four GG steps in the middle, which directly shows the difference in reactivity at the $3^{\prime}-G$ position for the three sequences used.

For DNA(1) in which the GG steps are in the AGGA sequence, the ratio of $5^{\prime}$ - to $3^{\prime}$-reactivity is $1.8 \pm 0.1$, and for $\mathrm{DNA}(2)$, which contains the sequence TGGT, this ratio is $6.1 \pm 0.3$. Thus, as previously reported, ${ }^{6,17}$ the relative reactivity of the $3^{\prime}-\mathrm{G}$ of GG steps decreases ca. 3-fold when embedded in a TGGT sequence compared with an AGGA sequence. Critically, the experiments reveal that for the U-containing sequence, $\operatorname{DNA}(3)$, the ratio of $5^{\prime}$ to $3^{\prime}$-reactivity of the GG steps is $3.4 \pm 0.2$, which is intermediate between the TGGT and AGGA sequences. The observed pattern of reaction selectivity for the XGGX sequences correlates well with our finding that the water access distribution functions are sequence invariant for the $5^{\prime}-\mathrm{G}$, but differ for the $3^{\prime}-\mathrm{G}$. Clearly, electronic factors assessed by ionization energies or the hole distributions, which are essentially sequence invariant (Table 1), do not appear to be the primary factor determining relative reactivity of guanines in GG steps. Instead, the accessibility of $\mathrm{H}_{2} \mathrm{O}$ to the reaction site determined by steric blocking by the thymine methyl group plays the dominant role for these sequences. ${ }^{18}$

In earlier work ${ }^{12}$ we showed that reaction of $\mathrm{H}_{2} \mathrm{O}$ with a guanine radical cation in an AGGA sequence has two enabling features: activation by association with a $\mathrm{Na}^{+}$ion and product stabilization by a nearby phosphate group. The results reported here reveal a third important feature-accessibility of reactants $\left(\mathrm{H}_{2} \mathrm{O}\right)$ to the reaction site, which is sequence-dependent and governed by steric effects. These finding may have implications to oxidative damage in cells where DNA is in a complex molecular environment.

Acknowledgment. We are grateful for support from the Air Force Office of Scientific Research (AFOSR), the U.S. Department of Energy (DOE), the National Science Foundation, and the Vassar Woolley Foundation. We thank Jianping Gao for assistance.

Supporting Information Available: General experimental methods, calculated ionization potentials, phosphorimagery data, X-ray structural data showing the stacking in TGGT and UGGU sequences, and structural characterization data for the oligonucleotides. This material is available free of charge via the Internet at http://pubs.acs.org.

\section{References}

(1) Schuster, G. B.; Landman, U. Topics Curr. Chem. 2004, 236, 139-162.

(2) Wagenknecht, H. A. Nat. Prod. Rep. 2006, 23, 973-1006.

(3) Joseph, J.; Schuster, G. B. J. Am. Chem. Soc. 2006, 121, 6070-6074.

(4) Joy, A.; Ghosh, A. K.; Schuster, G. B. J. Am. Chem. Soc. 2006, 128 $5346-5347$

(5) Hall, D. B.; Holmlin, R. E.; Barton, J. K. Nature 1996, 382, 731-735.

(6) Nakatani, K.; Dohno, C.; Saito, I. J. Am. Chem. Soc. 1999, 121, 1085410855 .

(7) Spassky, A.; Angelov, D. Biochemistry 1997, 36, 6571-6576.

(8) Sugiyama, H.; Saito, I. J. Am. Chem. Soc. 1996, 118, 7063-7068.

(9) Voityuk, A. A.; Jortner, J.; Bixon, M.; Rosch, N. Chem. Phys. Lett. 2000, 324, 430-434. However, see also: Voityuk, A. A. J. Phys. Chem. B 2005 , 109, 10793-10796.

(10) Prat, F.; Houk, K. N.; Foote, C. S. J. Am. Chem. Soc. 1998, 120, 845846.

(11) Senthilkumar, K.; Grozema, F. C.; Guerra Fensca, C.; Bickelhaupt, F. M.; Siebbeles, L. D. A. J. Am. Chem. Soc. 2003, 125, 13658-13659.

(12) Barnett, R. N.; Bongiorno, A.; Cleveland, C. L.; Joy, A.; Landman, U.; Schuster, G. B. J. Am. Chem. Soc. 2006, 128, 10795-10800.

(13) See Supporting Information for details.

(14) The geometry of the stacked quartets was constructed using the standard global helical parameters of B-DNA. These gas phase calculations included the sugar-phosphate groups and six $\mathrm{Na}^{+}$counter ions (three on each strand) located near the phosphate groups. The calculations used density functional theory (spin-density functional for the radical cations) with the generalized gradient approximation (GGA) [Perdew, J. P.; Burke, K.; Ernzerhof, M Phys. Rev. Lett. 1996, 77, 3865-3868.] and norm conserving soft pseudopotentials. [Troullier, N.; Martins, J. L. Phys. Rev. B 1991, 43 1993-2006.] A plane-wave basis (cut-off energy of 68 Ry) was used, with no supercell periodic replication of the ions, thus enabling accurate treatment of charged systems: Barnett, R. N.; Landman, U. Phys. Rev. B 1993, 48, 2081-2097.

(15) The MD simulations were carried out at $T=300 \mathrm{~K}$, for $10 \mathrm{~ns}$ each with a time step of $1 \mathrm{fs}$, using the Amber 96 potentials: Cornell, W. D.; Cieplak, P.; Bayly, C. I.; Gould, I. R.; Merz, K. M.; Ferguson, D. M.; Spellmeyer D. C.; Fox, T.; Caldwell, J. W.; Kollman, P. A. J. Am. Chem. Soc. 1995 , 117, 5179-5197. The partial charges of uracil where taken as those fitted for simulations of RNA. TIP3P potentials [Jorgensen, W. L.; Chandrasekhar, J.; Madura, J. D.; Impey, R. W.; Klein, M. L. J. Chem. Phys. $183,79,926$.] were used for water. No cutoffs were used for either the electrostatic or Lennard-Jones interactions.

(16) The samples were irradiated for $10 \mathrm{~min}$ (single hit conditions) at $350 \mathrm{~nm}$, where only AQ absorbs, in air-saturated sodium phosphate buffer solution $(\mathrm{pH} 7)$ at ambient temperature. In the quantitative analyses of relative reactivity, interference caused by end effects was eliminated by considering only the four central "identical" GG steps, but including the others does not change the conclusion.

(17) Liu, C.-S.; Hernandez, R.; Schuster, G. B. J. Am. Chem. Soc. 2004, 126, 2877-2884.

(18) While the dynamically fluctuating hydrating environment and counterion distribution may influence the IP values and hole distributions (e.g., hole localization) [Barnett, R. N.; Cleveland, C. L.; Joy, A.; Landman, U.; Schuster, G. B. Science 2001, 294, 567-571.], they are likely to remain largely sequence independent, leaving our conclusions unchanged.

JA071893Z 